\title{
Relationship between Microalbuminuria and Disease Activity in Patients with Ulcerative Colitis
}

\author{
Kourosh Masnadi Shirazi ${ }^{1}$, Sima Khayati ${ }^{2}$, Maryam Baradaran Binazir ${ }^{3}$, Zeinab Nikniaz ${ }^{1, *}$
}

1. Liver and Gastrointestinal Diseases Research Center, Tabriz University of Medical Sciences, Tabriz, Iran

2. Student Research Committee, Tabriz University of Medical Sciences, Tabriz, Iran

3. Medical Education Research Center, Health Management and Safety Promotion Research Institute, Tabriz University of Medical Sciences, Tabriz, Iran

\section{* Corresponding Author:}

Zeinab Nikniaz, MD

Liver and Gastrointestinal Diseases Research Center, Tabriz University of Medical Sciences, Tabriz, Iran Telefax : + 984133367499

Email: nikniazz@tbzmed.ac.ir

Received: 20 Jul. 2019

Accepted: 11 Dec. 2019

\section{ABSTRACT}

\section{BACKGROUND}

Introducing a non-invasive method for determining disease activity is important in patients with ulcerative colitis (UC). So in this study, we aimed to assess the association between disease activity index and microalbuminuria in patients with UC.

\section{METHODS}

In the present cross-sectional study, 84 patients with UC were selected. The disease activity was calculated by the partial Mayo clinic score. Microalbuminuria was assessed using the immunoturbidimetric method in a first-voided sample in the morning in two consecutive days and the mean of these two measurements was reported as urinary microalbumin level. Serum $\mathrm{C}$ reactive protein (CRP), erythrocyte sedimentation rate (ESR), and fecal calprotectin were measured respectively using conventional turbidimetric immunoassay, Westergren method, and ELISA methods.

\section{RESULTS}

The mean age of the participants was $40.01 \pm 12.85$ years, $60.8 \%$ of them were female and $53.5 \%$ had microalbuminuria. The frequency of microalbuminuria was significantly higher in patients with active compared with inactive inflammatory bowel disease (IBD). There were significant differences between the patients with active and inactive disease regarding CRP, ESR, and calprotectin $(p<0.001)$. Moreover, there was a strong correlation between microalbuminuria and CRP $(\mathrm{r}=0.89, p<0.001)$, ESR $(\mathrm{r}=0.92, p<0.001)$, and calprotectin $(\mathrm{r}=0.91, p<0.001)$.

\section{CONCLUSION}

Microalbuminuria could be used as a non-invasive marker of disease activity in patients with UC.

\section{KEYWORDS:}

Microalbuminuria; Ulcerative colitis; C-reactive protein; Calprotectin

\section{Please cite this paper as:}

Masnadi Shirazi K, Khayati S, Baradaran Binazir M, Nikniaz Z. Relationship between Microalbuminuria and Disease Activity in Patients with Ulcerative Colitis. Middle East $J$ Dig Dis 2020;12:34-38. doi: 10.15171/mejdd.2020.161.

\section{INTRODUCTION}

Ulcerative colitis (UC) is a chronic condition that is manifested by recurrent episodes of inflammation in the gastrointestinal tract. ${ }^{1}$ It is punctuated by periods of relapse and remission and the activity of the disease can be classified as mild, moderate, and severe. ${ }^{2}$ Disease severity influences the treatment choices in patients with UC. ${ }^{3}$ So, determining disease severity is important during the course 
of the disease. However, its assessment remains a difficult challenge. The most reliable method requires endoscopy with biopsy sampling, which is an invasive diagnostic tool. So different non-invasive laboratory parameters including CRP, ESR, and fecal calprotectin have been studied to predict disease severity and inflammation in such patients. ${ }^{4}$ Microalbuminuria occurs as a result of acute-phase response to inflammatory mediators and has previously been used as a marker of vascular dysfunction and the progression of kidney disease. ${ }^{5}$ Moreover, it has been suggested as a marker of disease severity in rheumatoid arthritis. ${ }^{6}$ Few studies also suggested using microalbuminuria as a disease activity index in inflammatory bowel disease (IBD). Mahmud and colleagues studied microalbuminuria in 42 patients with Crohn's disease and ulcerative colitis and showed a strong correlation between microalbuminuria and the intestinal histopathological grading system. ${ }^{7}$ In another study on 95 patients with IBD (52 patients with UC and 43 with Crohn's disease), a strong association was observed between microalbuminuria and index of Harvey and Bradshaw. ${ }^{8}$ On the other hand, Derici and co-workers did not show a significant difference between patients with active disease and the patients in remission regarding urine albumin levels. ${ }^{9}$

Considering the importance of identification of noninvasive laboratory parameters to predict disease severity and inflammation and strong correlation between microalbuminuria and other laboratory markers of disease activity such as erythrocyte sedimentation rate (ESR) and C reactive protein (CRP) and the limited number of studies that assessed the association between microalbuminuria and disease activity index in patients with UC, we aimed to assess the association between disease activity index and microalbuminuria in patients with UC.

\section{MATERIALS AND METHODS}

\section{Subject selection}

In the present cross-sectional study, 84 patients with UC who were diagnosed by gastroenterologists in Inflammatory Bowel Disease Clinic of Imam Reza Hospital affiliated to Tabriz University of Medical Sciences, Tabriz-Iran in 2017 were selected using convenience sampling. The patients were included if they were diagnosed as having UC and aged 18-85 years. The patients who had other disorders that are associated with microalbuminuria including pyelonephritis, glomerulonephritis, diabetes, amyloidosis, nephrotic syndrome, lupus, and renal vein thrombosis were excluded from the study.

The sample size was calculated based on a $95 \%$ confidence interval, $80 \%$ power, and considering the expected 0.9 standardized differences in microalbuminuria level between studied groups, which led to 62 cases. Considering the attrition rate of $30 \%$, the total sample size was assumed 84 patients ( 42 patients in remission and 42 patients with active UC).

Written informed consent was obtained from all patients. The Ethics Committee of Tabriz University of Medical Sciences approved the study (Ethics code:IR.TBZMED. REC. 1396.1127)

The partial Mayo clinic score was used for UC activity. This score is based on stool frequency, rectal bleeding, and the physician's rating of disease activity. Each part was scored between 0 and 3 points. The total UC activity score was obtained by summing these three items (0-9). The patients were considered as having active UC if their partial Mayo score was more than 4. Moreover, according to the partial Mayo score, the patients were classified as having remission (Mayo score $<2$ ), $\mathrm{mild} /$ moderate disease severity (Mayo score 2-7), and severe disease activity (Mayo score $>7$ ).

Microalbuminuria was assessed using the immunoturbidimetric method in a first-voided sample in the morning in two consecutive days and the mean of these two measurements was reported as urinary albumin level. Albuminuria was categorized based on the National Renal Association Criteria. Serum CRP, ESR, and serum calprotectin were measured respectively using conventional turbidimetric immunoassay, Westergren method, and ELISA method.

\section{Statistical analysis:}

In the present study, the data distribution was analyzed by Kolmogorov-Smirnov test. The continuous variables were presented as mean and standard deviation (SD) and the categorical variables were presented as frequency (\%). Independent $t$ test and Chi-square test were used for between-groups comparison. The one-way ANOVA with Tukey post-hoc tests were used for comparison of urine albumin level between different categories 
Table 1: Baseline characteristics of the participants

\begin{tabular}{lcccc}
\hline Variables & Total & $\begin{array}{c}\text { Active } \\
(\mathbf{n = 4 2})\end{array}$ & $\begin{array}{c}\text { Inactive } \\
(\mathbf{n}=\mathbf{4 2})\end{array}$ & $\boldsymbol{p}$ value* \\
\hline Age (years) & $40.01 \pm 12.85$ & $39.21 \pm 12.32$ & $4085 \pm 13.48$ & 0.56 \\
\hline Sex & & & \\
\hline Male & $33(39.2 \%)$ & $16(38.09 \%)$ & $17(40.47 \%)$ & 0.82 \\
\hline Female & $51(60.8 \%)$ & $26(61.90 \%)$ & $25(59.52 \%)$ & \\
\hline Microalbuminuria & & & \\
\hline Positive & $39(53.5 \%)$ & $42(100 \%)$ & $3(7.14 \%)$ & 0.001 \\
\hline Negative & $39(46.5 \%)$ & $0(0 \%)$ & $39(92.85 \%)$ & \\
\hline Extraintestinal manifestations & & & \\
\hline Positive & $2(24.8 \%)$ & $13(30.95 \%)$ & $7(16.66 \%)$ & \\
\hline Negative & $64(76.2 \%)$ & $29(69.04 \%)$ & $35(83.33 \%)$ & \\
\hline * $p$ value of independent t test & &
\end{tabular}

of disease severity. Pearson correlation coefficient test was used for assessing the correlation between microalbuminuria and CRP, ESR, and calprotectin. A probability level of 0.05 was chosen for statistical significance. All analyses were done by SPSS software version 22 .

\section{RESULTS}

Table 1 shows the participants' characteristics. The mean age of the participants was $40.01 \pm 12.85$ years, $60.8 \%$ of them were female and $53.5 \%$ had microalbuminuria. The frequency of microalbuminuria was significantly higher in patients with active disease compared with inactive IBD.

Table 2 presents comparisons of the laboratory parameters between the patients with active and inactive disease. As can be seen, there were significant differences between patients with active and inactive disease regarding all study parameters $(p<0.05)$ except for the urine creatinine $(p=0.44)$.

The comparison of the urine albumin level according to the disease severity of UC is presented in table 3 . According to the result of one-way ANOVA, there was a significant difference in urine albumin levels between different groups. Moreover, the result of Tukey post-hoc test also showed that the mean level of urine albumin was significantly higher in patients with severe disease compared with patients in mild/moderate and remission phase.

The association between microalbuminuria, CRP, ESR, and calprotectin level is shown in figure 1 . According to the results of the Pearson correlation coefficient test, there was a strong correlation between microalbuminuria and $\operatorname{CRP}(\mathrm{r}=0.89, p<0.001), \operatorname{ESR}(\mathrm{r}=$ $0.92, p<0.001)$, and calprotectin $(\mathrm{r}=0.91, p<0.001)$.

\section{DISCUSSION}

In the present study, the frequency of microalbuminuria in patients with UC and also its correlation with disease activity was assessed. According to the results, 53\% of the patients in the present study had microalbuminuria. Previously, Herrlinger and colleagues reported that 52\% of the patients with IBD had proteinuria. ${ }^{10}$ Babayeva and colleagues also found albuminuria in $61.5 \%$ of patients with UC. ${ }^{11}$ However, Mahmud and co-workers reported that $100 \%$ of patients with IBD had microalbuminuria. ${ }^{8}$ The observed differences between the reports of different studies may be related to the differences in the forms of the diseases (UC Vs Crohn's disease or both), the selected marker of proteinuria (microalbuminuria vs. albuminuria and $\alpha^{1}$-microglobulin- $\alpha^{1}-\mathrm{MG}$ ) and the type of drugs used by patients.

In line with the result of a previous study, ${ }^{7}$ there is a significant association between microalbuminuria and CRP level in patients with UC. However, in the study by Derici and colleagues, there was no significant association between albuminuria and CRP level. ${ }^{9}$ Moreover, Herrlinger did not either report any association between proteinuria and CRP in patients with IBD.10 The differences in the results of studies may be due to the differences in inclusion criteria (UC vs. CD and UC) and type of administered drugs.

In the present study, we showed a significant association between microalbuminuria and disease activity in patients with UC. Similar result was published by Mahmud and colleagues. Moreover, Herringer and others also reported a strong association between proteinuria and disease activity in patients with IBD. ${ }^{10}$ Derici and colleagues reported the significant differences in urine albumin levels between patients with active UC compared with the control group, however, the differences between patients with active disease and the patients in remission were not statistically significant. ${ }^{9}$ The differences between the results of various studies may be due to the differences in the type of administered drugs, disease activity, and inflammation status in patients. The proposed underlying mechanisms for the association between microalbuminuria and 
Table 2: Comparison of the laboratory findings between active and inactive ulcerative colitis

\begin{tabular}{|c|c|c|c|c|}
\hline Variables & $\begin{array}{c}\text { Total } \\
(\text { Mean } \pm \text { SD })\end{array}$ & $\begin{array}{l}\text { Active patients } \\
\text { (Mean } \pm \text { SD) }\end{array}$ & $\begin{array}{c}\text { Inactive patients } \\
(\text { Mean } \pm \text { SD })\end{array}$ & $p$ value* \\
\hline CRP (ng/L) & $3.00 \pm 2.77$ & $5.065 \pm 1.07$ & $0.35 \pm 0.28$ & $<0.001$ \\
\hline $\mathrm{ESR}(\mathrm{mm} / \mathrm{hr})$ & $35.58 \pm 32.26$ & $653.85 \pm 14.89$ & $5.30 \pm 2.84$ & $<0.001$ \\
\hline Urinary albumin (mg/L) & $103.41 \pm 107.44$ & $195.76 \pm 75.70$ & $11.07 \pm 12.86$ & $<0.001$ \\
\hline Urine creatinine (mg/dL) & $948.87 \pm 195.49$ & $965.095 \pm 192.16$ & $932.26 \pm 260.30$ & 0.44 \\
\hline Serum calprotectin (ng/ml) & $330.10 \pm 368.66$ & $648.16 \pm 260.30$ & $12.04 \pm 10.88$ & $<0.001$ \\
\hline
\end{tabular}

${ }^{*} p$ value of independent $t$ test; CRP: C-Reactive protein; ESR: Erythrocytes sedimentation rate

Table 3: Comparison of the urine albumin level according to disease severity of ulcerative colitis

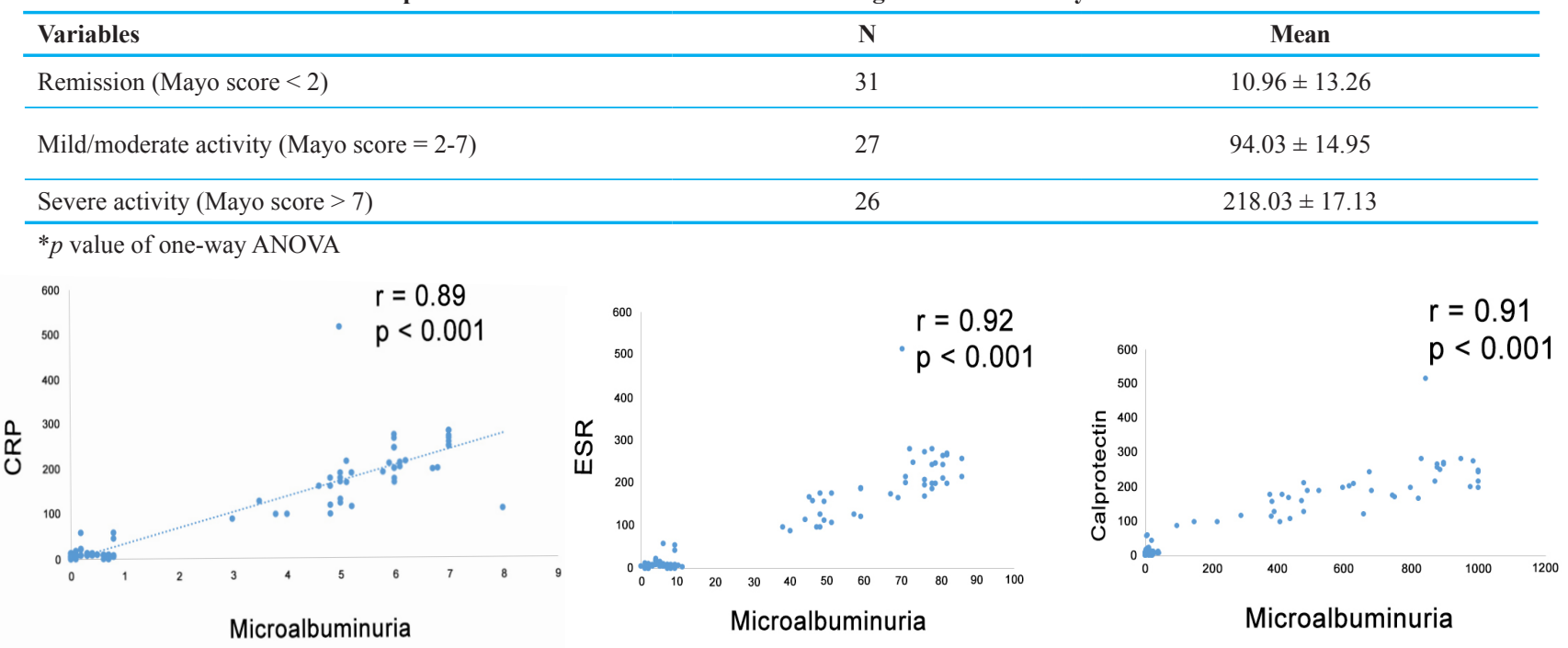

Fig.1: The association between microalbuminuria and C-reactive protein, erythrocytes sedimentation rate, and calprotectin in patients with ulcerative colitis

disease activity in patients with UC could be related to an increase in inflammatory cytokines in patients with active UC. These cytokines could disrupt mucosal sulphated glycosaminoglycans in the renal microvasculature that increases vascular permeability for albumin..$^{12,13}$

The results of this study suffer from some limitations. In the present study, the disease activity was measured using a partial Mayo clinic score. Although, it is a valid method of measuring disease activity in patients with UC, applying more valid methods that consider histological grading of the disease activity could provide more precise results. Moreover, in the present study, we did not consider the effect of different drugs on microalbuminuria level and also the sample size was limited.

In conclusion, the results of the present study showed a significant association between microalbuminuria and disease activity in patients with UC. From the clinical point of view, the finding of the present study indicated that microalbuminuria could be used as a non-invasive marker of disease activity in patients with UC. However, for precise results, there is a need for a longitudinal and large scale study considering the confounding factors.

\section{ACKNOWLEDGMENT}

The authors wish to thank the Liver and Gastrointestinal Diseases Research Center, Tabriz University of Medical Sciences for financial support. The results of the present study are derived from the Sima Khayati thesis in general medicine.

\section{ETHICAL APPROVAL}

There is nothing to be declared. 
CONFLICT OF INTEREST

The authors declare no conflict of interest related to this work.

\section{REFERENCES}

1. Loddo I, Romano C. Inflammatory bowel disease: genetics, epigenetics, and pathogenesis. Front Immunol 2015;6:551. doi: 10.3389/fimmu.2015.00551.

2. Gracie DJ, Ford AC. Evidence-based management of ulcerative colitis. Minerva Gastroenterol Dietol 2012;58:87-99.

3. Meier J, Sturm A. Current treatment of ulcerative colitis. World J Gastroenterol 2011;17:3204 -12. doi: 10.3748/ wjg.v17.i27.3204.

4. Walsh AJ, Bryant RV, Travis SP. Current best practice for disease activity assessment in IBD. Nat Rev Gastroenterol Hepatol 2016;13:567-79. doi: 10.1038/nrgastro.2016.128. Epub 2016 Sep 1.

5. Glassock RJ. Is the presence of microalbuminuria a relevant marker of kidney disease? Curr Hypertens Rep 2010;12:364-8. doi: 10.1007/s11906-010-0133-3.

6. Niederstadt C, Happ T, Tatsis E, Schnabel A, Steinhoff J. Glomerular and tubular proteinuria as markers of nephropathy in rheumatoid arthritis. Rheumatology $(O x-$ ford) 1999;38:28-33. doi: 10.1093/rheumatology/38.1.28.

7. Mahmud N, McDonald G, Kelleher D, Weir D. Microalbuminuria correlates with intestinal histopathological grading in patients with inflammatory bowel disease. Gut 1996;38:99-103. doi: 10.1136/gut.38.1.99

8. Mahmud N, Stinson J, O'Connell MA, Mantle TJ, Keeling PW, Feely J, et al. Microalbuminuria in inflammatory bowel disease. Gut 1994;35:1599-604. doi: 10.1136/gut.35.11.1599.

9. Derici U, Tuncer C, Ebinç FA, Mutluay R, Yakaryilmaz F, Kulaksizoglu S, et al. Does the urinary excretion of a1-microglobulin and albumin predict clinical disease activity in ulcerative colitis? Adv Ther 2008;25:1342-52. doi: 10.1007/s12325-008-0109-8.

10. Herrlinger K, Noftz M, Fellermann K, Schmidt K, Steinhoff $\mathrm{J}$, Stange E. Minimal renal dysfunction in inflammatory bowel disease is related to disease activity but not to 5-ASA use. Aliment Pharmacol Ther 2001;15:363-9. doi: 10.1046/j.1365-2036.2001.00940.x.

11. Babayeva G, Babayev Z. Frequency of detection of some markers of endothelial dysfunction in patients with inflammatory bowel diseases. Ter Arkh 2018;90:12-6. doi: 10.26442/terarkh201890412-16.

12. Klein NJ, Shennan GI, Heyderman RS, Levin M. Alteration in glycosaminoglycan metabolism and surface charge on human umbilical vein endothelial cells induced by cytokines, endotoxin and neutrophils. J Cell Sci 1992;102:821-32.

13. Murch SH, MacDonald TT, Walker-Smith JA, Lionetti P, Levin M, Klein NJ. Disruption of sulphated glycosaminoglycans in intestinal inflammation. Lancet 1993;341:711-4. doi: 10.1016/0140-6736(93)90485-y. 